\title{
MODELOS PARA LA DATACIÓN DE HIERROS Y ACEROS ANTIGUOS APLICADOS A TIZONA
}

\author{
POR \\ JoSÉ MANUEl JiMÉNEZ, DANIEL ARIAS, ESTHER BRAVO, \\ JUAN ANTONIO MARTÍNEZ y ANTONIO J. CRIADO
}

\section{RESUMEN - ABSTRACT}

El presente artículo trata sobre el estudio de la valoración del envejecimiento natural de piezas arqueológicas, como modelo para la datación de piezas de hierro y acero antiguas. También se indican otros tres métodos a modo de introducción, uno es del Carbono 14, y los otros se basan en la difusión de elementos a través de la capa de corrosión y a través de escorias. El método estudiado se ha aplicado al caso concreto de la espada Tizona proponiendo un método matemático que nos permite averiguar su antigüedad. Para este estudio nos hemos ayudado de técnicas como la Microscopía Óptica y Microscopía Electrónica de Barrido (MEB), tanto de baja como de alta resolución.

This article deals with the study of the assessment of the natural aging of archaeological pieces, as a dating model for ancient pieces of iron and steels. As introduction of the article, it also shows other three methods. The first one is radiocarbon dating. The other ones are based on the diffusion of some elements by means of the corrosion layer and the slags. The method, which has been researched, has been applied to the specific issue of the sword Tizona. It propounds a mathematical method, which allow us to verify its age. Optic Microscopy and Scanning Electron Microscopy (SEM), both low and high resolution, are techniques used to carry out this study.

\section{PALABRAS CLAVE - KEY WORDS}

Tizona. Datación. Envejecimiento natural. Carburos de hierro. Difusión.

Tizona. Datation. Natural aging. Iron carbides. Difussion.

\section{INTRODUCCIÓN}

Hasta hoy la posible datación de una pieza de hierro o acero, se venía haciendo a partir de su tipología y de la aparente tecnología aplicada durante su fabricación por conformación plástica y posterior acabado. Hay casos en los que esta sistemática de datación ofrece cierta fiabilidad, pero en otros casos, esto nos lleva a la incertidumbre.

Hemos puesto en marcha varios modelos para tratar de valorar la antigüedad de una pieza arqueológica en hierros y aceros. Cada modelo es aplicable según el historial de la pieza estudiada.

En ocasiones, los modelos son complementarios unos de otros, pero otras veces, sólo es aplicable el más adecuado. Clasificándolos de una manera simple, nuestras propuestas van en las siguientes direcciones:

1. Valoración mediante Carbono-14. 
2. Valoración de la difusión de Si y de Fe en partículas de cuarzo ocluidas en la superficie exterior de la capa de corrosión.

3. Valoración de la difusión del Fe y el Si en partículas de escorias ocluidas en el material.

4. Valoración del envejecimiento estructural natural.

\subsection{Valoración mediante Carbono-14}

En el primer método se trata de calcular la relación isotópica del Carbono contenido en el acero o fundición. Según esa relación isotópica, se pueden ver las cantidades relativas de los isótopos de este elemento, y por tanto, del $\mathrm{C}^{14}$. Es muy fiable ya que la presencia de este $\mathrm{C}^{14}$ no se ve alterada por los años en que la pieza arqueológica estuvo expuesta a contaminaciones del medio ambiente. El análisis se realiza mediante ICP Masas y con una precisión notable, siendo su parte más complicada la preparación de la muestra para el análisis, que ha hecho que este método se encuentre aún en su fase de desarrollo en la Universidad de Oxford.

1.2. Valoración de la difusión de Si y de Fe en partículas de cuarzo ocluidas en la superficie exterior de la capa de corrosión

El segundo método puede ser aplicado cuando la pieza metálica ha permanecido enterrada. El inicio de la formación de la primera capa de óxidos de hierro es traumática con respecto al terreno que envuelve a la pieza. Suele acarrear el aprisionamiento de partículas del terreno a causa del aumento de volumen de esta capa y por la presión ejercida por el terreno que la envuelve.

A continuación, y debido al contacto íntimo entre estas partículas asimiladas y los óxidos de hierro, comienzan los procesos de difusión. Estos procesos de difusión de silicio en los cristales de óxidos de hierro y de hierro en los de cuarzo pueden ser cuantificados y asociados al tiempo de contacto gracias a la Microsonda Electrónica. Eso sí, solamente se puede datar con respecto al tiempo que lleva enterrada. Es un procedimiento muy útil para la arqueología, pero no en otros casos de piezas arqueológicas que no hayan sufrido este proceso.

Estas piezas arqueológicas que han permanecido enterradas durante largos periodos de tiempo presentan una morfología común, observándose siempre tres zonas bien diferenciadas:

- Zona más interna, constituida por el núcleo metálico, de tamaño variable en función del proceso de corrosión sufrido por la pieza.

- Zona intermedia constituida por óxidos de hierro compactos, en el que no aparecen inclusiones. Estos óxidos de hierro pueden ser de diferente naturaleza, aunque en la gran mayoría de los casos están formados por magnetita $\left(\mathrm{Fe}_{3} \mathrm{O}_{4}\right)$.

- Zona externa, con estructura fibrosa en la que aparecen gran número de partículas e inclusiones procedentes del medio circundante. Esta capa de óxido hace que el óxido de hierro, que ocupa más volumen, tienda a expandirse, atrapando las ya mencionadas partículas minerales del entorno.

Para determinar la composición química de las piezas arqueológicas, tanto de su núcleo metálico como de los óxidos formados, se procede a realizar análisis puntuales utilizando la técnica de Microsonda Electrónica, observándose, que en el óxido de hierro que se encuentra próximo a una inclusión silicatada la concentración de silicio es irregularmente alta.

Analizando una línea, en la que tomamos como punto de partida el interior de uno de estos silicatos, y como punto final un punto dentro del óxido de hierro, bien alejado del borde 
del silicato, se obtiene una curva de tipo sigmoidal al representar la concentración de silicio frente a la distancia, (muy similar a las obtenidas en procesos de difusión, utilizando el modelo de Matano).

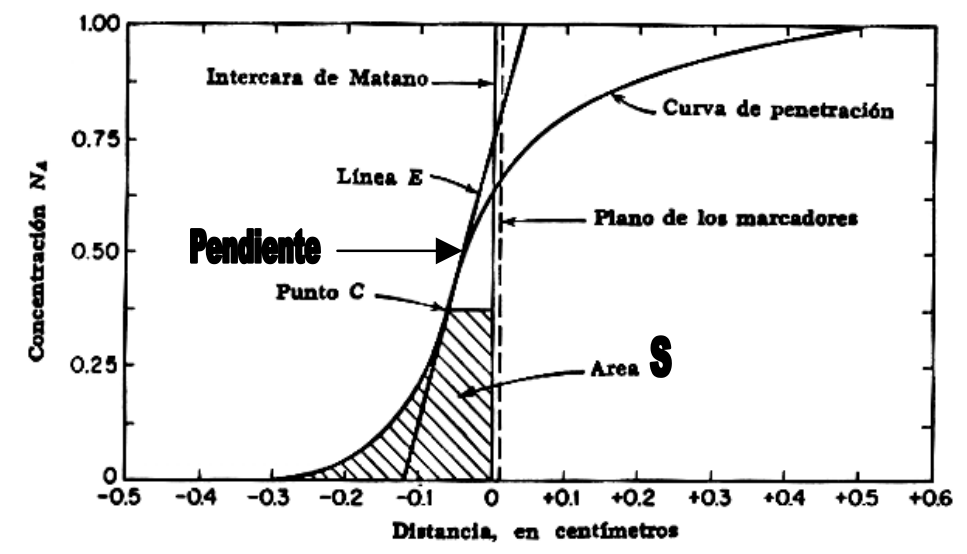

Según el modelo de Matano, los datos se ajustan a la expresión:

$$
D^{*}=\frac{1}{2 \cdot t} \cdot K \cdot S[1]
$$

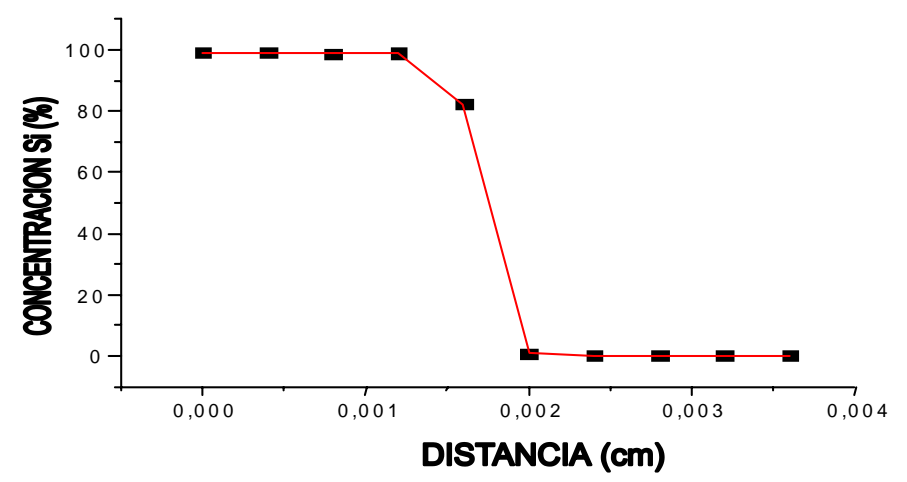

Concentración de silicio-distancia según el modelo de Matano.

La curva resultante es una sigmoidal.

Siendo: $\left\{\begin{array}{l}D^{*}=\text { difusividad } \\ t=\text { tiempo de difusión } \\ K=\text { inversa de la pendiente de la curva en un punto dado } \\ S=\text { área de la curva que se encuentra entre el punto y la denominada intercara } \\ \text { de Matano. }\end{array}\right.$ 


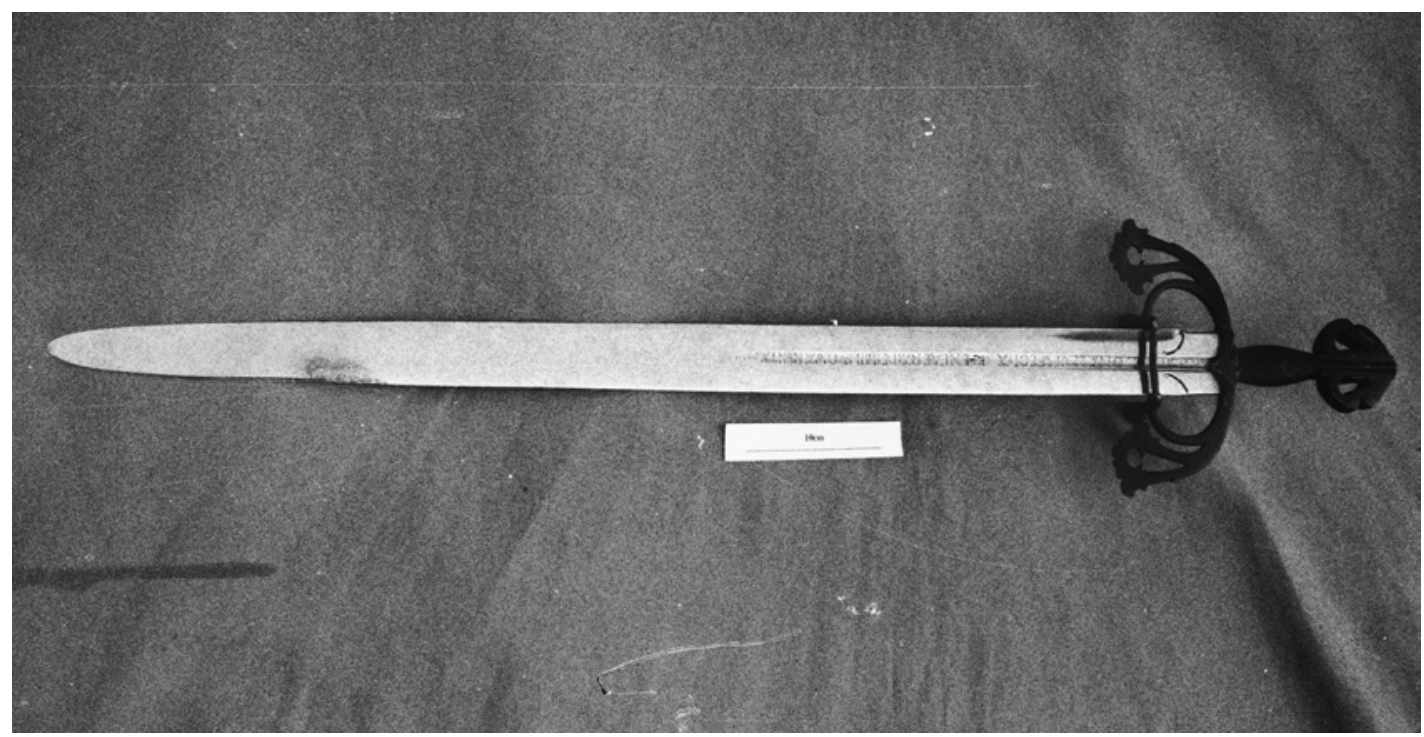

Fig. 1. Tizona, la legendaria espada del Cid Campeador. Se trata de una hoja de acero andalusí, tipo jineta o tajadora, útil para el combate a caballo.

La difusividad es una variable que depende de la concentración de forma polinómica, según una expresión de segundo grado:

$$
y=a \cdot x^{2}+b \cdot x+c[2]
$$

Representando la difusividad en función de $K \cdot S$ obtendremos una línea cuya pendiente es. $1 / 2 t$ Los resultados cosechados incluyen un error inferior al $5 \%$, siendo los tiempos estimados suficientemente significativos y fiables.

\subsection{Valoración de la difusión del Fe y el Si en partículas de escorias ocluidas en el material}

El tercer método viene a complementar al anteriormente descrito. En él, no va a depender en su año de conteo o datación cero el hecho de haber permanecido enterrada o no la pieza.

En este caso, como en el segundo método, se trata de valorar la difusión producida entre la matriz metálica y las escorias ocluidas durante el proceso de forja en caliente. Se mide, por tanto, la penetración del silicio en la matriz de ferrita, y la del hierro en la partícula de escoria, formada generalmente, por silicatos complejos. Es necesario conocer la naturaleza de la escoria para poder definir el coeficiente de difusión del hierro en el silicato. Frente al segundo método, presenta ventajas: la no contaminación con el entorno y, que el proceso difusivo, comienza en el momento de su conformación plástica (Fig. 2)

\subsection{Valoración del envejecimiento estructural natural}

El cuarto método, que es el que presentamos en esta publicación aplicado a Tizona, supone valorar mediante metalografía cuantitativa el cambio microestructural producido por envejecimiento natural. 


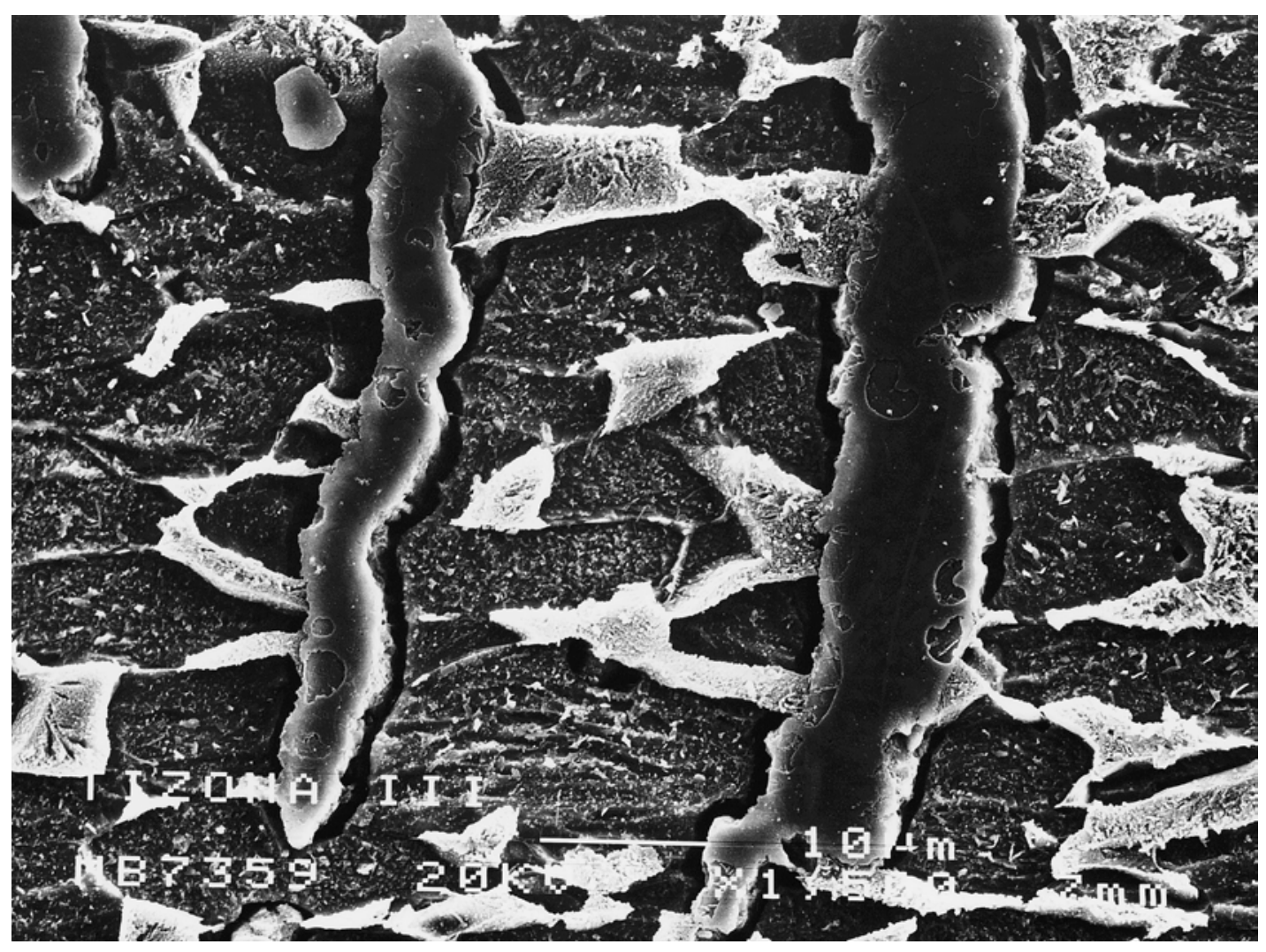

Fig. 2. Escorias de Tizona, incluidas en el acero, paralelas a la superficie.

Tizona (Fig. 1), era la espada favorita del héroe legendario Don Rodrigo Díaz, el Cid Campeador, que nació en Vivar (provincia de Burgos) en fecha incierta, entre los años 1043 y 1048, y murió en Valencia en 1099 (Fletcher 1990). A lo largo de la historia Tizona ha sufrido serias manipulaciones. La tosca empuñadura, que presenta en la actualidad, pertenece a los siglos XV-XVI, siendo las inscripciones latinas realizadas al ácido, algo anteriores a esta fecha. Este hecho ha provocado que numerosos especialistas, basándose en su tipología, consideraran que la actual Tizona es una falsificación muy posterior al siglo XI.

Esta investigación ha ido dirigida a valorar el proceso de envejecimiento estructural del acero de la espada. Nuestra experiencia en arqueometalurgia de aceros antiguos (Criado et alii, 1999. Criado et alii, 2000), nos ha permitido desarrollar un modelo matemático, que permitiese una datación de este arma legendaria. La herramienta fundamental para este estudio ha sido la Metalografía Cuantitativa del cambio estructural del acero de Tizona, debida al envejecimiento con el paso de los siglos. Para ello, se ha empleado como técnica, la Microscopía Electrónica de Barrido de Alta Resolución.

\section{TÉCNICA EXPERIMENTAL}

Dado que el origen, o tiempo cero, para valorar la evolución estructural por envejecimiento natural, coincide con el último tratamiento termomecánico llevado a cabo en la pieza a datar, se seleccionaron muestras de aquellas zonas de la hoja que con seguridad no habían sufrido ningún tratamiento termomecánico posterior a su forja y temple iniciales. 
Estas muestras, de pequeñísimo tamaño, se embutieron en resina para su correcta preparación metalográfica. Las superficies una vez pulidas, se atacaron con Nital al $2 \%$ durante 30 segundos. Finalmente se recubrieron con oro por sputtering para su correcta observación por Microscopía Electrónica de Barrido de Alta Resolución, seleccionando aquellas zonas en las que el cambio estructural por envejecimiento era más acusado, con el fin de poder cuantificarlo.

\section{DisCUSIÓN DE RESULTADOS}

Los aceros, al igual que otros materiales, cambian su estructura con el paso del tiempo, debido a un proceso difusivo de envejecimiento estructural lento a temperatura ambiente ${ }^{2-3}$. Este cambio estructural favorece la formación de estructuras más relajadas tensionalmente, con mayor perfección cristalina, y por tanto más estables.

La naturaleza de este cambio está relacionada con la morfología inicial de las fases producidas en los procesos termomecánicos de conformación plástica de las piezas. Los cristales de ferrita, perlita, bainita y martensita, evolucionan a estructuras morfológicas diferentes.

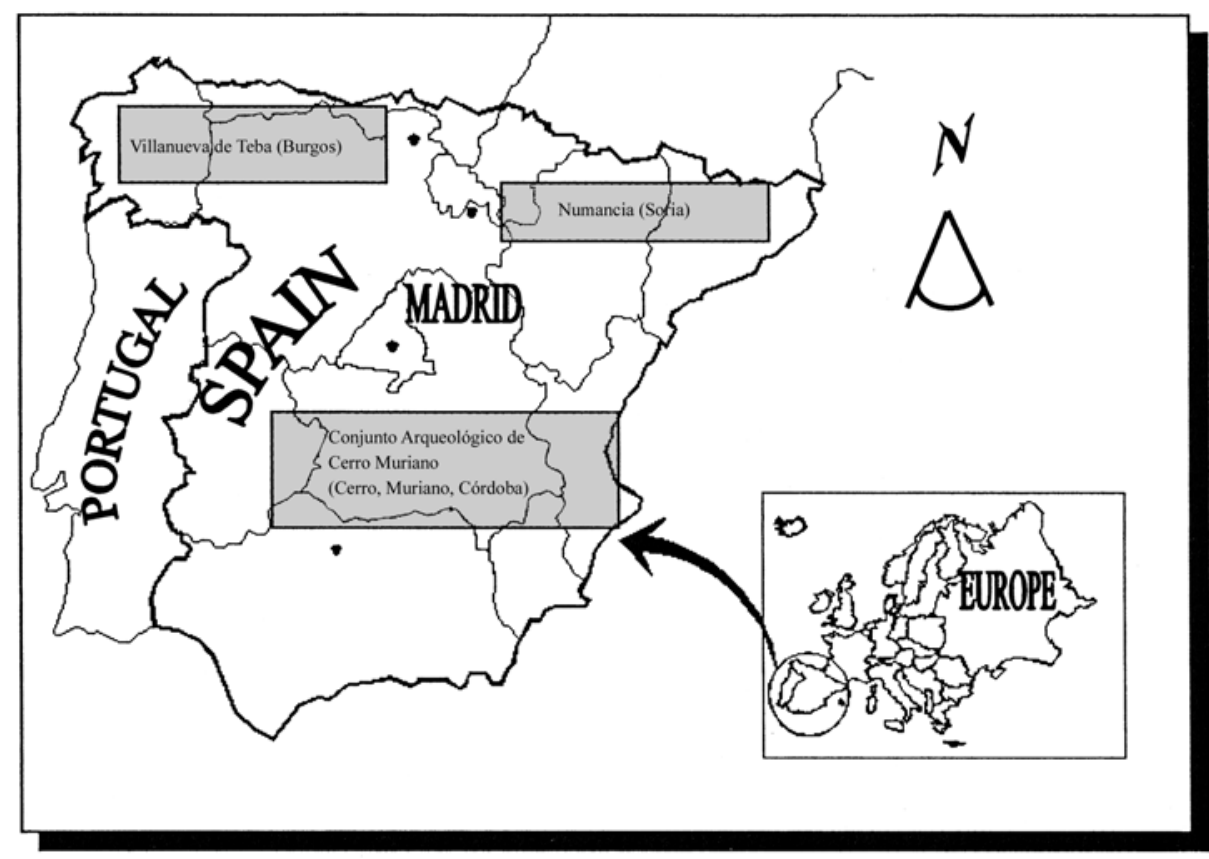

Fig. 3. Mapa geográfico de la Península Ibérica con la localización de los yacimientos arqueológicos citados en este trabajo.

La ferrita basa su cambio estructural en la segregación del carbono que tiene en exceso, dada la baja solubilidad de éste en hierro $\alpha$ a bajas temperaturas, como se observa en el acero de una tachuela de la Necrópolis Celtibérica de Numancia (Figs. 3 y 4). Para la perlita, el cambio está basado en la formación de cristales prismáticos idiomórficos de cementita de notable paralelismo, como se puede ver claramente en el acero hipereutectoide de un clavo de la Villa Romana Altoimperial del Procurator Metallorum de Cerro Muriano (Córdoba) (Figs. 3 y 5). La formación de estas estructuras se debe al perfeccionamiento cristalino y la 


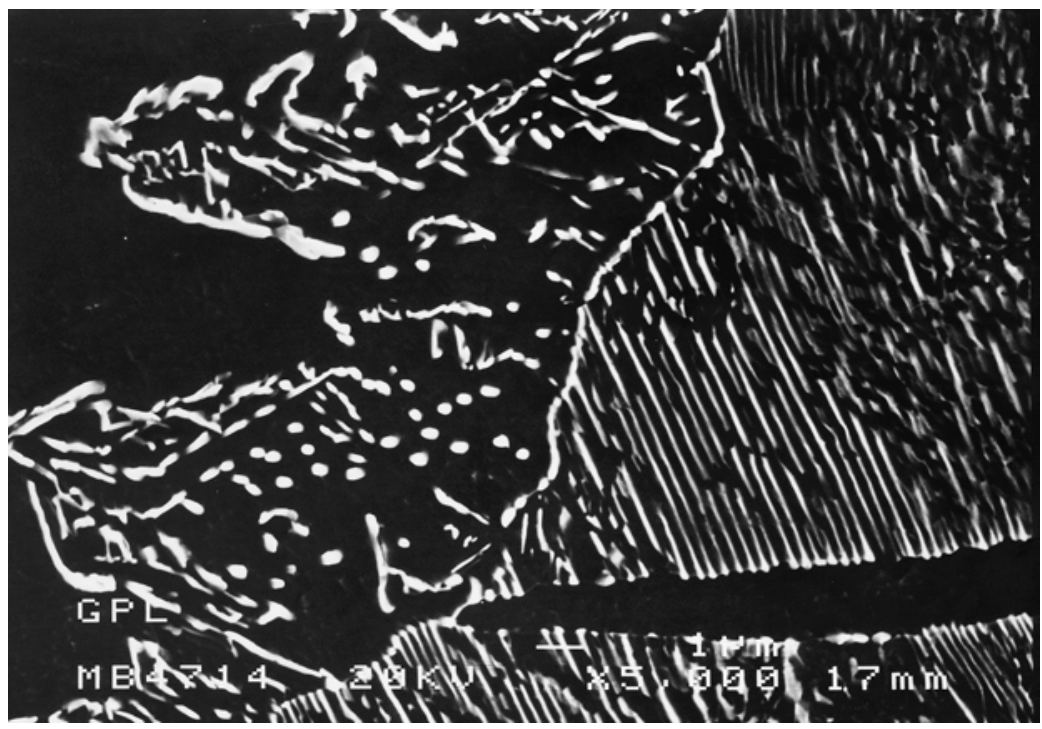

Fig. 4. Micrografía en donde se muestra la ruptura de las láminas de cementita presentes en la ferrita, y la posterior esferoidización

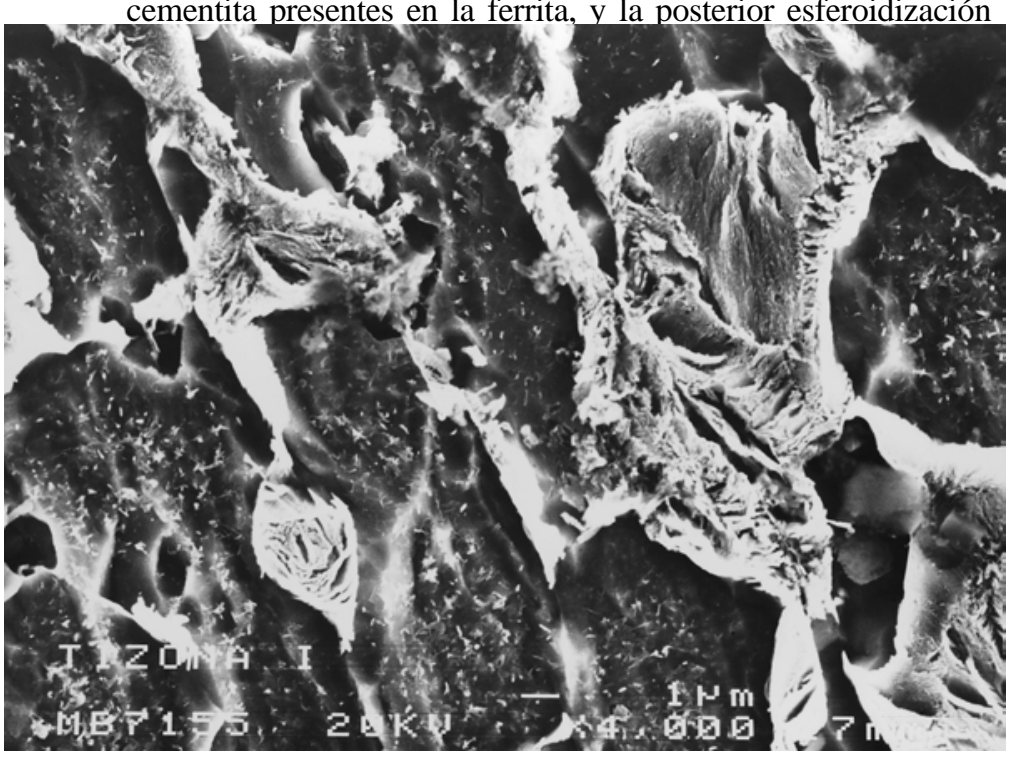

Fig. 5. Formación de placas prismáticas idiomórficas de cementita en un clavo de acero hipoeutectoide de las Termas Altoimperiales de Cerro Muriano, (Córdoba), perteneciente al siglo I d. C.

relajación de tensiones por escalonamiento y crecimiento de cristales. En el caso de estructuras como bainitas y martensitas, el proceso se ve favorecido por la relajación interna en los cristales, a la vez que se produce una segregación y crecimiento de carburos de hierro esferoidizados con morfologías arracimadas. 


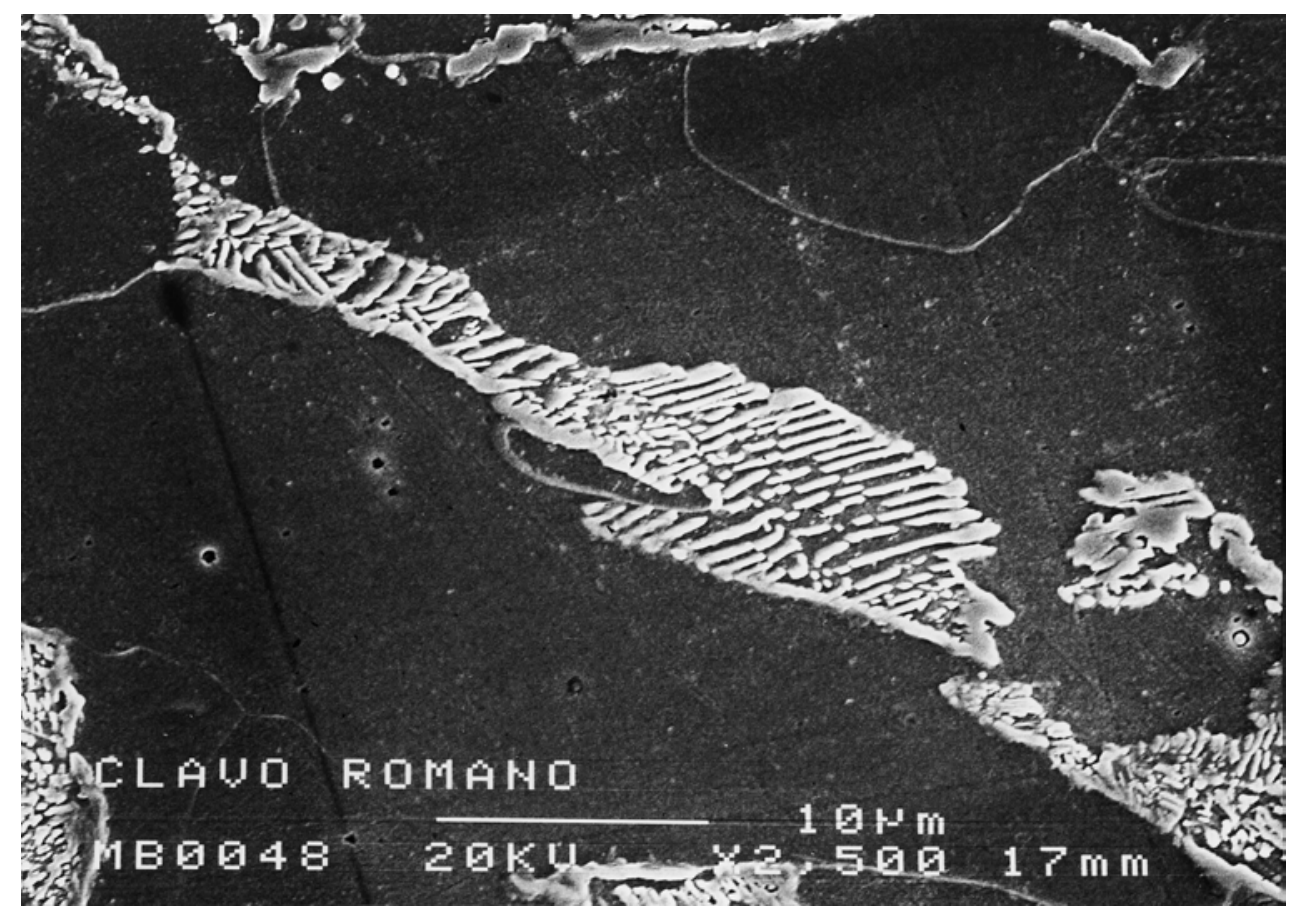

Fig. 6. El acero del núcleo de la hoja acerada de Tizona mostrando colonias de perlita y cristales de ferrita.

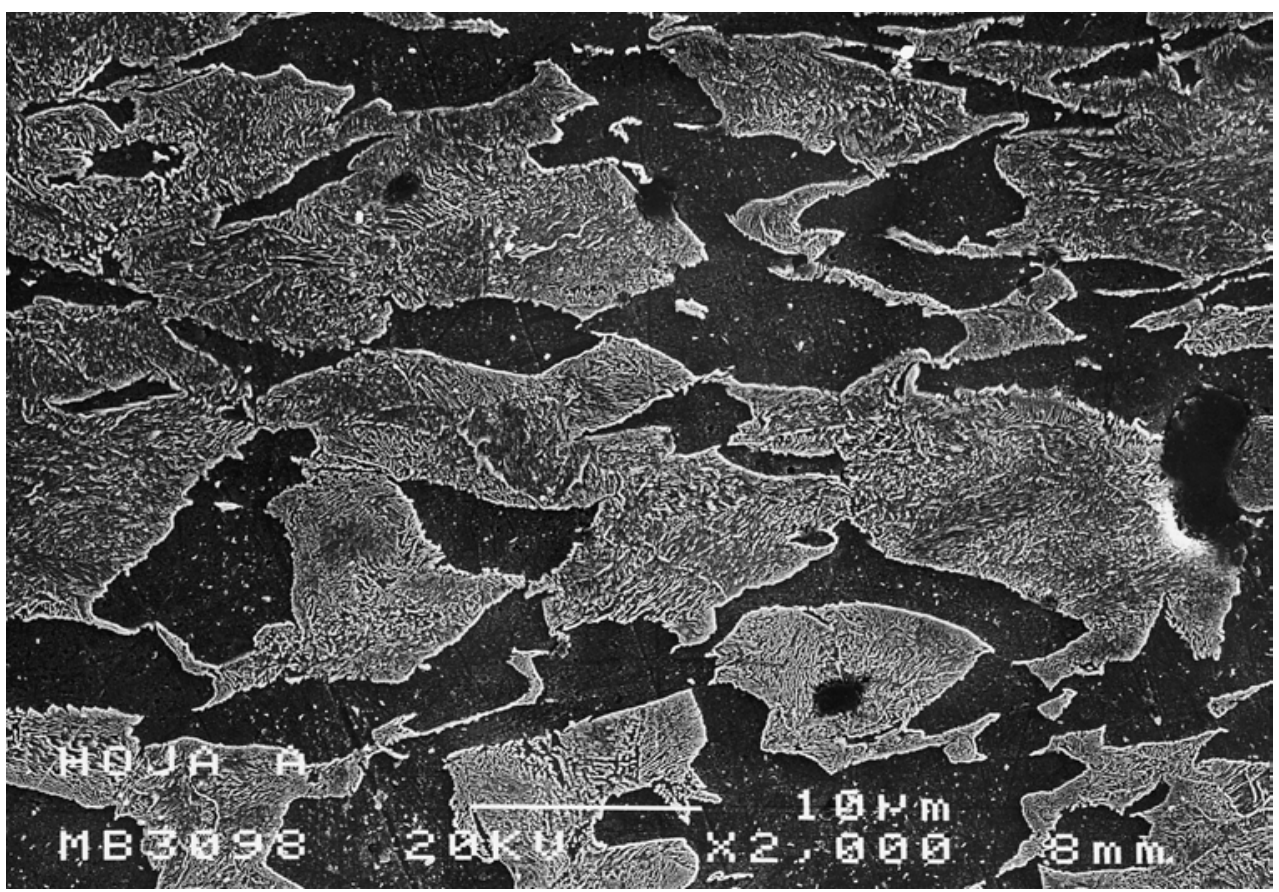

Fig. 7. Finos cristales de carburo de hierro precipitados en la matriz ferrítica del núcleo de la espada Tizona. 


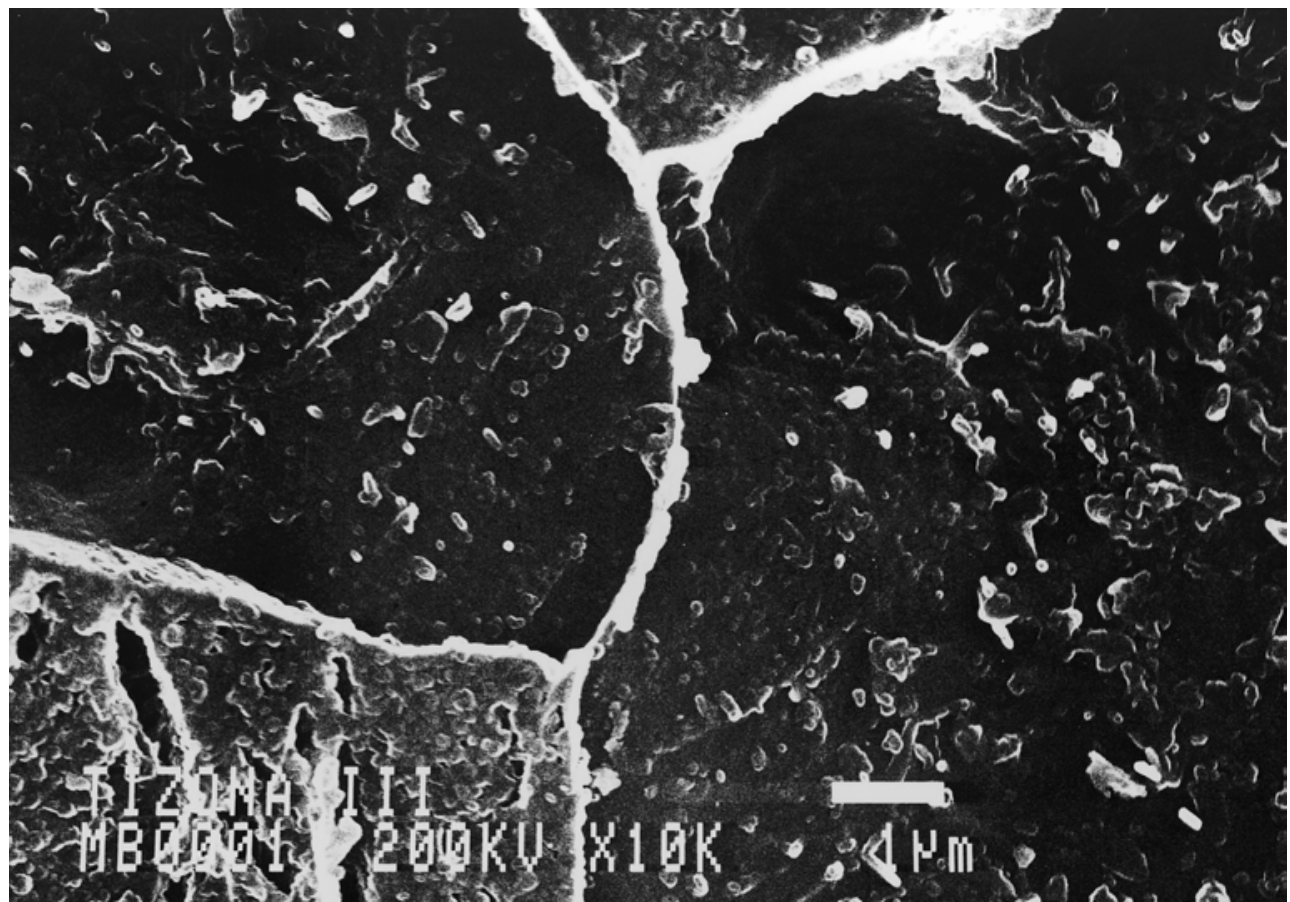

Fig. 8. En esta figura se observa la distribución de estos finos cristales de carburo de hierro precipitados en ferrita en el acero de Tizona.

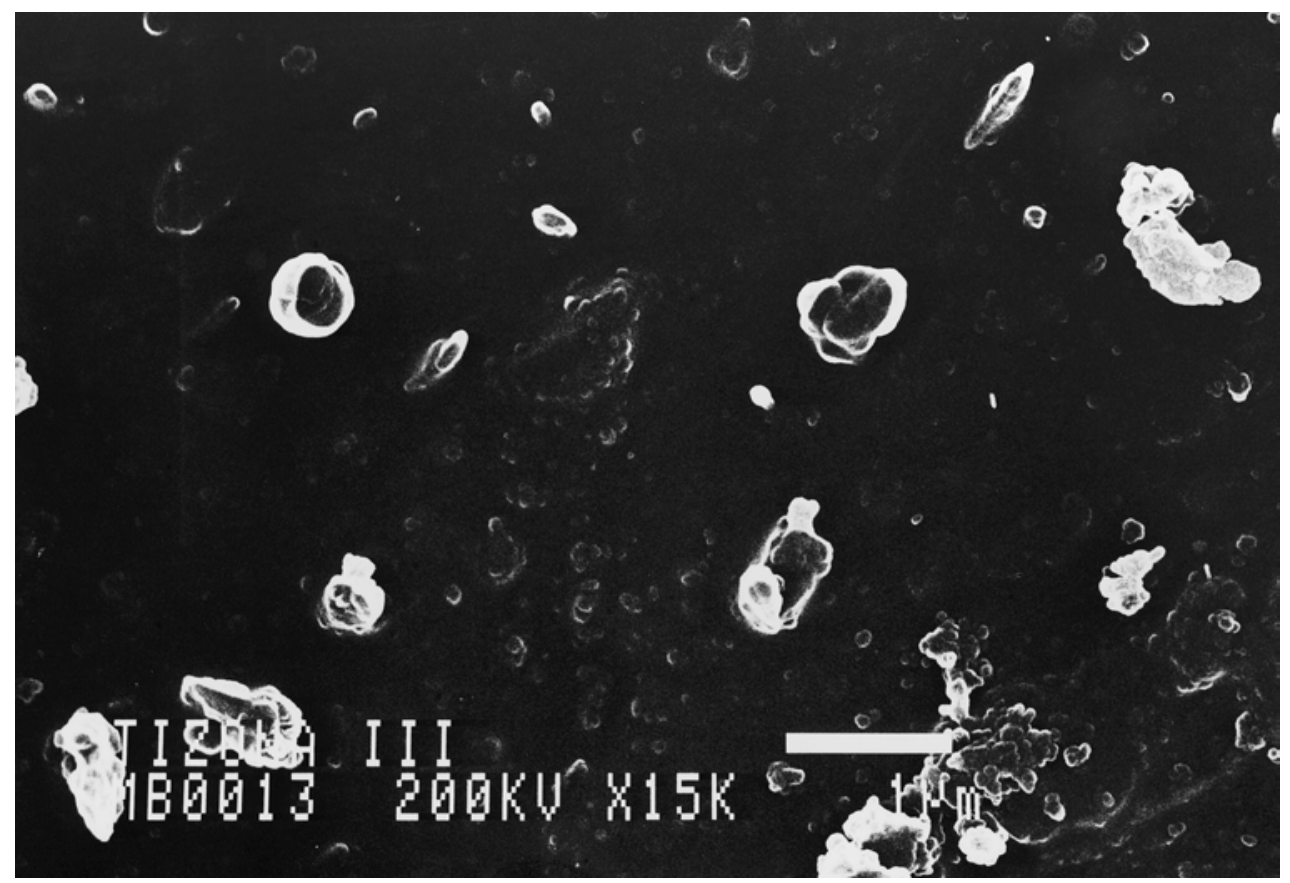

Fig. 9. Detalle de la figura anterior, mostrando la morfología de los cristales de carburo de hierro y la diferencia de tamaño. 


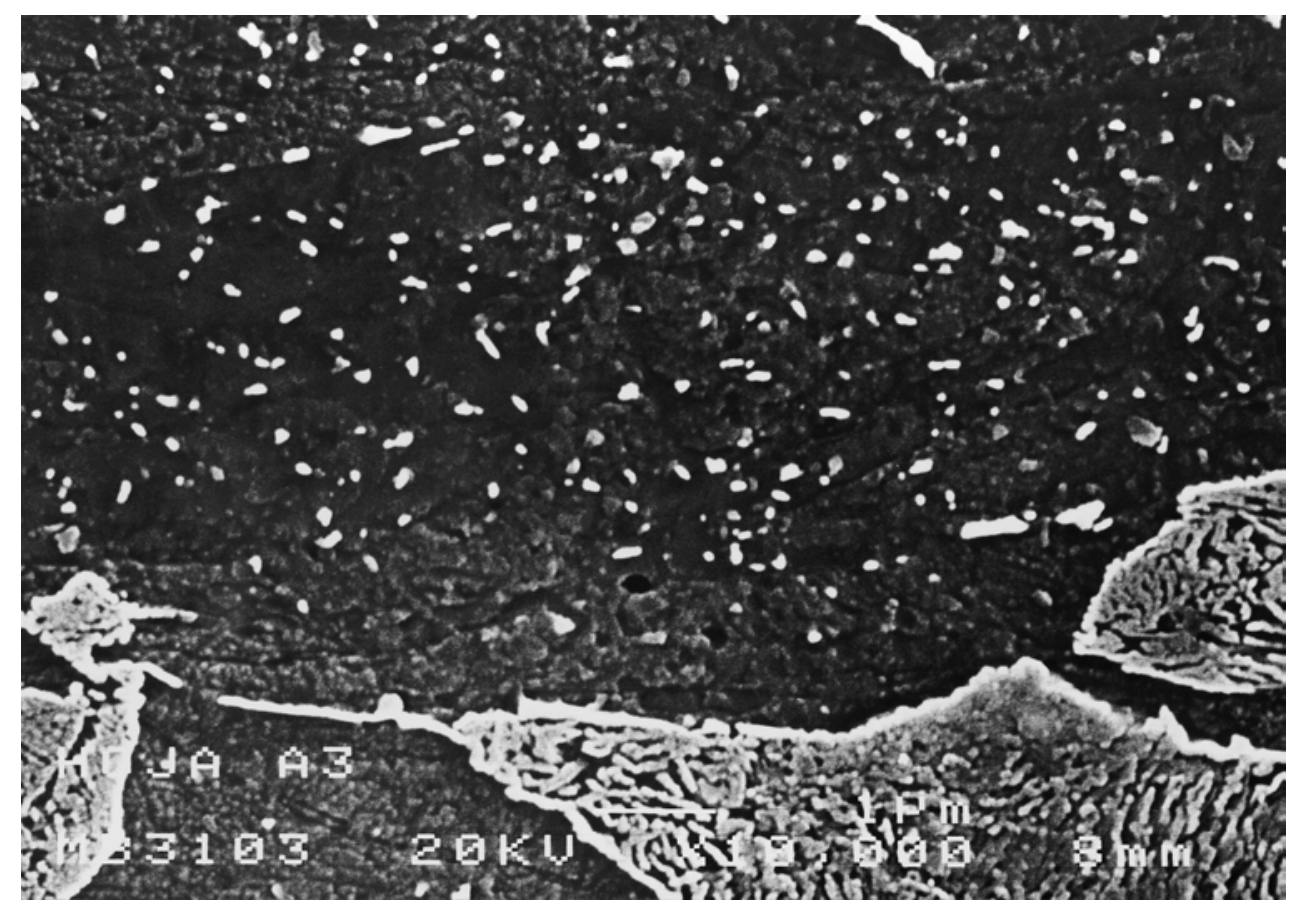

Fig. 10. Detalle de la figura 7 en donde se observa los momentos iniciales en la precipitación de los cristales de carburo de hierro.

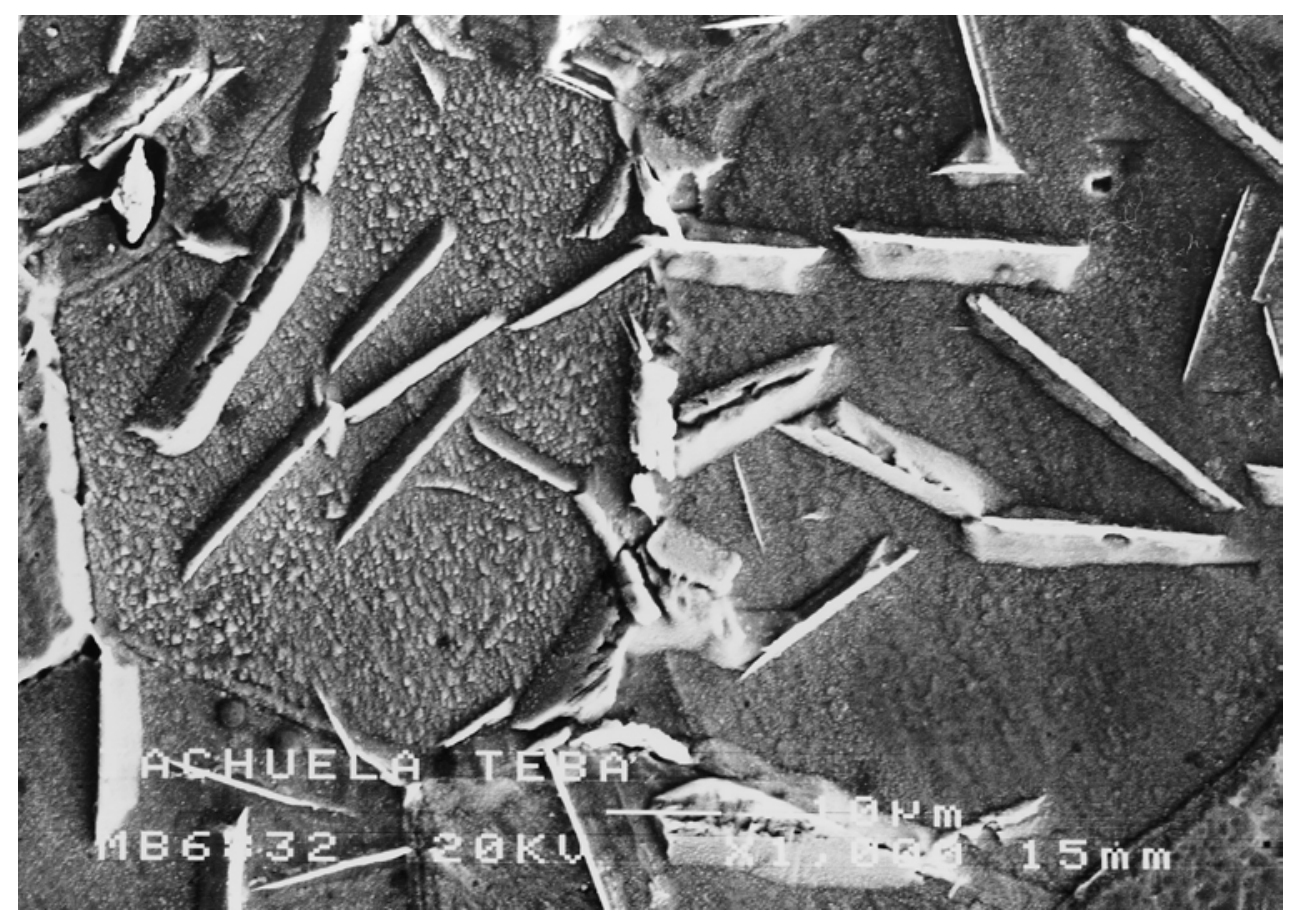

Fig. 11. Cristales de carburo de hierro precipitados en ferrita en una tachuela, de la Necrópolis Ibérica de Villanueva de Teba (Burgos), perteneciente al siglo IV a.C. 
En la hoja acerada de Tizona se suceden todos los procesos anteriormente mencionados, seleccionando de todos ellos la segregación de carburos de hierro en los cristales de ferrita por su fácil visualización e interpretación metalúrgica. Dado que la hoja de Tizona está compuesta por dos aceros distintos: uno con un elevado contenido en carbono en la zona externa, y otro de muy bajo contenido en su interior (Criado et alii, 2000b), elegimos éste último para el estudio.

La energía necesaria para que se dé el proceso de envejecimiento estructural lento, es debida a la deformación plástica residual acumulada y a las tensiones internas creadas por la contracción de volumen durante el enfriamiento, hecho que se ve favorecido por la saturación en carbono de la ferrita $\left(0,025 \% \mathrm{C}\right.$ a $723^{\circ} \mathrm{C}$ y $0,008 \%$ C a $0^{\circ} \mathrm{C}$ - en peso-), ocurrido tanto para un tratamiento de normalizado tras la forja en caliente, como por un proceso de temple.

El acero del interior de la hoja de Tizona, presenta colonias perlíticas y cristales de ferrita (Fig. 6). Estos cristales de ferrita muestran un fino precipitado de carburos de hierro, que se han generado por la segregación del carbono de la matriz ferrítica (Fig. 7). En las figuras 8, 9 y 10 se observa el tamaño, distribución y morfología de estos cristales de carburo de hierro. El tamaño es muy variable para casos como el de Tizona, que sólo cuenta con unos 950 años de antigüedad. Para otros aceros con cronologías comprendidas entre 1500 y 2500 años, el tamaño de los carburos de hierro es menos variable, como se observa en el acero hipereutectoide de una tachuela de la Necrópolis Ibérica de Villanueva de Teba (Burgos), (Figs. 3 y 11).

La medida precisa del diámetro medio de los cristales de carburo de hierro precipitados en la matriz ferrítica, es el dato experimental necesario para el cálculo de la datación del acero.

Se ha propuesto un modelo matemático (Porter y Easterling, 1981) para medir el tiempo de la hoja de Tizona, basándose en los cambios microestructurales por envejecimiento. Este modelo es válido desde pocos siglos, hasta 2500 años, y parte de la siguiente ecuación:

$$
f\left(e_{m}^{3}\right)=K \cdot t[3]
$$

en donde $\boldsymbol{e}_{\boldsymbol{m}}$ es el tamaño medio de las partículas, $\boldsymbol{K}$ es una constante, y $\boldsymbol{t}$ es el tiempo en segundos. La constante $\boldsymbol{K}$, depende a su vez de:

$$
K=D \cdot X_{e} \cdot \gamma[4]
$$

siendo $\boldsymbol{D}$, el coeficiente de difusión del carbono en la ferrita a temperatura ambiente, $\boldsymbol{X}_{\boldsymbol{e}}$ la concentración de carbono en el equilibrio dentro de la fase ferrita y $\gamma$ la energía interfacial.

Dando valores a cada una de las variables, y partiendo de otros aceros, perfectamente datados y conocidos, se obtiene un valor para la constante $K$ de:

$$
K=135,50 \cdot 10^{-33}\left(\mathrm{~m}^{3} / \mathrm{s}\right)
$$

Si lo aplicamos al caso concreto de Tizona, se obtiene un diámetro medio de $\overline{e_{m}}=1,5952 \cdot 10^{-7}(\mathrm{~m})=0,1595(\mu \mathrm{m})$, que una vez introducido en la ecuación, resulta:

$$
\begin{aligned}
& e_{m}{ }^{3}=135,50 \cdot 10^{-33}\left(\mathrm{~m}^{3} / \mathrm{s}\right) \cdot t(s)[5] \\
& t(s)=\frac{e_{m}{ }^{3}\left(m^{3}\right)}{135,50 \cdot 10^{-33}\left(\mathrm{~m}^{3} / \mathrm{s}\right)}[6]
\end{aligned}
$$




$$
\begin{aligned}
& t(s)=\frac{4,056 \cdot 10^{-21}\left(\mathrm{~m}^{3}\right)}{135,50 \cdot 10^{-33}\left(\mathrm{~m}^{3} / \mathrm{s}\right)}[7] \\
& t(s)=2,9959 \cdot 10^{10}(\mathrm{~s})[8] \\
& t(\text { años })=950(\text { años })[9]
\end{aligned}
$$

De los cálculos realizados, se deduce que el acero de Tizona se fabricó y forjó hace pues, aproximadamente, 950 años, lo que la sitúa en la época del legendario Cid Campeador.

\section{CONCLUSIONES}

El envejecimiento natural de hierros y aceros antiguos provoca cambios microestructurales notables, en los que las morfologías finales de las fases dependen de la estructura de partida y del tiempo transcurrido.

La medida del tamaño de los cristales de carburo de hierro, segregados en la ferrita, permite cuantificar el cambio microestructural de la hoja de acero de Tizona.

Utilizando el modelo matemático definido en la ecuación $f\left(e_{m}{ }^{3}\right)=K \cdot t$, se ha podido datar a Tizona como forjada hace, aproximadamente, 950 años.

Nota: Este estudio ha sido realizado en el Instituto de Microscopía Lluis Bru de la Universidad Complutense de Madrid.

José MAnuel JimÉnez, DANiel Arias, Esther Bravo, JuAn Antonio Martínez y ANTONio J. CRiado

Dpto. de Ciencia de los Materiales e Ingeniería Metalúrgica. Facultad de CC. Químicas. Universidad Complutense de Madrid.

\section{BIBLIOGRAFÍA}

FLeTCHER, R. (1989): El Cid. Editorial Nerea, Madrid,.

Criado A. J.; Martínez, J. A.; García, J. I. y Ruíz J. (1999): «Origin of El Cid's Sword Revealed by ICM-MS Metals Analysis». Spectroscopy Europe: 11/4.

CRiado, A. J.; Martínez, J.; CAlabrés, R.; Rodríguez, L. M.; JimÉnez, J. M. y KarlsSon M. (2000): «Microstructures in Historical and Archaeological Steel Objects Resulting from Aging Processes». Praktiche Metallographie: 37, 6.

Criado, A. J.; Martínez, J. A.; García, J. I.; Ruíz, J. (2000b): «Metallographic Study of the Steel Blade of the Sword Tizona». Praktische Metallographie: 37, 7.

PORTER, D. A. and EASTERLING, K. E. (1981): Phase Transformations in Metals and Alloys. Ed. Van Nostrand Reinhold, New York. 